\title{
Aproximación al conocimiento, actitud y práctica en salud reproductiva de perros machos con propietario
}

\author{
Approach to the knowledge, attitude and practice in reproductive health \\ of male dogs with owner
}

\author{
Alfonso Sánchez R. ${ }^{1,2}$, Mariela Pfeffer P. ${ }^{1}$
}

\section{Resumen}

\begin{abstract}
Considerando que la salud y el bienestar animal son quehaceres que no deben disociarse, si no, más bien, deben estar ligados para una mayor compresión y establecimiento de una salud integral, se presenta la importancia de establecer en este estudio pautas de salud reproductiva en perros adultos enteros, a través de evidencia médica, como así también investigar los parámetros que valoran el conocimiento, actitud y práctica en relación con la tenencia responsable. En el presente estudio, se realizaron encuestas como técnica de recolección de datos a 50 tenedores de perros. En el examen de salud reproductiva se reconocieron mayores frecuencias de anomalías subclínicas, prostáticas, y testiculares en perros mayores de 6 años, respecto de animales más jóvenes $(\mathrm{p}<0.05)$. Se observó aumento de tamaño prostático en el $75 \%$ de los perros. Con respecto al conocimiento y la actitud en tenencia responsable de mascotas y salud reproductiva, las respuestas evidenciaron estar de acuerdo con las buenas prácticas, sin embargo, en el quehacer diario no se vio plenamente reflejado.
\end{abstract}

Palabras clave: salud reproductiva, tenencia responsable, conocimiento, actitud

\footnotetext{
${ }^{1}$ Núcleo de Investigaciones Aplicadas en Ciencias Veterinarias y Agronómicas, Universidad de Las Américas (UDLA), Campus Los Castaños, Viña del Mar, Chile

${ }^{2}$ E-mail: asanchez@udla.cl
}

Trabajo financiado por el proyecto interno 2018028 de la Dirección de Investigación de la Universidad de las Américas.

Recibido: 2 de febrero de 2021

Aceptado para publicación: 7 de agosto de 2021

Publicado: 22 de diciembre de 2021

CLos autores. Este artículo es publicado por la Rev Inv Vet Perú de la Facultad de Medicina Veterinaria, Universidad Nacional Mayor de San Marcos. Este es un artículo de acceso abierto, distribuido bajo los términos de la licencia Creative Commons Atribución 4.0 Internacional (CC BY 4.0) [https:// creativecommons.org/licenses/by/4.0/deed.es] que permite el uso, distribución y reproducción en cualquier medio, siempre que la obra original sea debidamente citada de su fuente original 
The animal health and welfare are tasks that should not be dissociated, and therefore they should be linked for a greater understanding and establishment of comprehensive health. Based on this, the importance of establishing reproductive health guidelines in intact adult dogs, through medical evidence, as well as investigating the parameters that value knowledge, attitude and practice in relation to responsible ownership. In the present study, surveys were conducted as a data collection technique to 50 dog keepers. In the reproductive health examination, higher frequencies of subclinical, prostate, and testicular abnormalities were identified in dogs older than 6 years of age as compared to younger animals $(\mathrm{p}<0.05)$. Prostate enlargement was observed in $75 \%$ of dogs. Regarding the knowledge and attitude in responsible pet ownership and reproductive health, the responses showed that they agreed with good practices, however, it was not fully reflected in daily activities.

Key words: reproductive health, responsible ownership, knowledge, attitude

\section{INTRODUCCIÓN}

En la sociedad actual, el trato que los seres humanos prodigan a los animales es una preocupación moral. Esta preocupación se ha visto plasmada en la legislación, a través de indicaciones que incentivan la tenencia responsable de mascotas, como es el caso de la Ley de Tenencia Responsable N..$^{\circ} 21.020$ que entró en vigor en Chile en 2017. En esta ley se define el concepto de Tenencia Responsable (TR) como las responsabilidades que contrae una persona al aceptar la tenencia de una mascota y que conlleva, entre las principales obligaciones, a identificarlo mediante microchip e inscribirlo ante la autoridad competente en un registro nacional de mascotas, brindarle albergue, alimentación y buen trato, procurando además los cuidados veterinarios indispensables para su bienestar y no someterlo a sufrimientos a lo largo de su vida (Ministerio de Salud, 2017).

La ciencia del bienestar animal evalúa los comportamientos, las emociones y la salud (Dawkins, 2007). En la actualidad, se puede evidenciar que la salud y el bienestar animal son elementos indisociables y, por ello, el bienestar animal debería considerarse como un elemento importante para incorporar en las deliberaciones sanitarias clásicas (Nicks y Vandenheede, 2014). Sin duda, un gran desafío para tenedores de mascotas caninas y médicos veterinarios, asociado a los cambios en la calidad de vida del mundo globalizado, es el incremento en la esperanza de vida de los perros (Villamil et al., 2008), lo cual genera retos significativos para crear condiciones apropiadas para la mantención de la salud y calidad de vida en concordancia con los principios del bienestar animal (CaronLormier et al., 2016).

Los trastornos en el aparato reproductor de los perros son comunes y varios de ellos asociados a la longevidad, los cuales se pueden disminuir con medidas preventivas adecuadas (Meyers-Wallen, 2012). Es así que proponer, desarrollar y socializar el concepto de salud reproductiva, especialmente en una fase preventiva, vendría a complementar lo declarado en cuanto a bienestar animal y tenencia responsable (Sánchez, 2018). Desde este mismo ángulo, la Organización Mundial de Sanidad Animal (OIE) afirma que las actividades de prevención de las enfermedades animales aportan beneficios considerables para el bienestar animal (OIE, 2013). 
Es desde esta mirada que surge la pregunta ¿Hay bienestar animal sin una prevención integral de patologías? Es ahí, donde se desea poner énfasis y escrudiñar nuevos conceptos que vayan en beneficio de esta temática, integrando la concepción de salud reproductiva y sus implicancias. En Chile son escasos los estudios sistemáticos con esta orientación, lo cual sería un aliciente para establecer pautas en salud reproductiva a fin de aportar a la mejora de la calidad de vida de los perros y educar al público general en la conveniencia de la esterilización de ejemplares sin propósitos reproductivos $y$, por otra parte, en el caso de animales de valor reproductivo, postular sugerencias a los médicos veterinarios, para valorar la importancia de los estudios preventivos del aparato reproductor (Sánchez, 2018; Sánchez y Pfeffer, 2020). Por tanto, el propósito del presente estudio fue obtener evidencia médica del estado de salud reproductiva de perros machos y de parámetros que estiman el conocimiento, actitud y práctica en salud reproductiva y tenencia responsable de perros adultos enteros como un aporte al bienestar animal.

\section{Materiales y Métodos}

En el marco del proyecto «Caracterización de salud reproductiva y tenencia responsable en caninos machos de Viña del Mar, Chile: Una propuesta integradora basada en la evidencia», se trabajó con encuestas a 50 tenedores como técnica de recolección de datos. Dada las características del trabajo, donde la participación era voluntaria, el muestreo fue por conveniencia, y para cumplir con la responsabilidad ética y social de la investigación, se les solicitó a los participantes su consentimiento a través de una carta aprobada por el comité de ética de la institución (UDLA). La encuesta fue validada por juicio de expertos.
El estudio se llevó acabo entre enero de 2018 y septiembre de 2019 en el Centro Veterinario de la Universidad de Las Américas en la ciudad de Viña del Mar, Chile. Una vez realizado el examen clínico reproductivo a los ejemplares, según lo descrito por Sánchez et al. (2019), se procedió a aplicar el cuestionario a los tenedores.

La encuesta fue realizada por médicos veterinarios y el cuestionario contó con preguntas cerradas y abiertas, las cuales consideraron aspectos sociodemográficos y conocimientos, actitudes y prácticas de los tenedores de perros machos enteros, en relación con la tenencia responsable y la salud reproductiva. También se consideró una caracterización general de los tenedores a través de preguntas tales como: ¿Cuál es la principal razón de la tenencia de su mascota?, ¿Hace cuánto tiempo tiene esta mascota?, ¿Cómo llegó a su poder esta mascota?, ¿Su mascota tiene implantado el microchip?, ¿Dónde vive esta mascota? y ¿Cuántas mascotas caninas tiene a su cargo? y defina su relación con la mascota (dueño, amo, responsable, tenedor, propietario, poseedor $\mathrm{u}$ otra).

Previo al análisis de los datos, las variables de respuesta libre fueron agrupadas mediante conceptos predeterminados. Asimismo, la variable edad fue categorizada en tres clases. Se realizó estadística descriptiva y asociaciones de variables categóricas. El análisis de las preguntas de respuesta libre consideró text mining, donde las palabras fueron estandarizadas para determinar frecuencia. La asociación entre variables categóricas se estudió mediante la prueba del Chi cuadrado de Pearson. Todas las pruebas estadísticas fueron realizadas con un $95 \%$ de confianza $(\mathrm{alfa}=0.05)$ y un valor crítico de $\mathrm{p}=0.05$. Los datos fueron analizados con el programa Stata 14. 


\section{Resultados}

\section{Salud Reproductiva}

Los perros evaluados $(n=50)$ tenían edades entre 1 y 12 años, siendo la edad promedio de $5.54 \pm 3.82$, distribuida de la siguiente manera: $<2$ años $(26 \%)$, e $\gg 2 \mathrm{y}<6$ años $(38 \%)$ $\mathrm{y}>6$ años (36\%). El peso promedio fue de $26.4 \pm 6.9 \mathrm{~kg}$. El $46 \%$ de los perros fueron mestizos, 24\% Bulldog Inglés, 10\% Bull Terrier, 10\% Bóxer, 6\% Poodle, 2\% Dogo Alemán y $2 \%$ Labrador.

En el examen de salud reproductiva se encontró una mayor frecuencia de tono testicular disminuido (flacidez) y asimetría testicular en perros $>6$ años respecto a los más jóvenes $(p<0.05)$. En la ecografía testicular, se observó mayor frecuencia de alteraciones de parénquima, especialmente heterogeneidad y presencia de focos anecoicos en perros $>6$ años $(p<0.05)$, en tanto que en la evaluación ecográfica de próstata destacó la mayor proporción de anomalías de ecotextura, asimetría lobular, contorno glandular irregular y presencia de focos anecoicos en perros $>6$ años $(p<0.05)$. Cabe destacar que se observó prostatomegalia en el $75 \%$ de los perros.

En la citología prepucial no se observaron diferencias entre los grupos etarios, registrándose predominio de células intermedias y moderada a abundante cantidad de neutrófilos en los frotis.

\section{Encuestas}

El estudio fue realizado encuestando a 50 personas con edades entre 20 y 72 años, de las cuales $58 \%$ fueron mujeres y $42 \%$ hombres. Las características sociodemográficas de la muestra se presentan en el Cuadro 1.

En la caracterización general de los tenedores destacaron las siguientes respuestas:
Cuadro 1. Características sociodemográficas de tenedores de perros machos enteros (Viña del Mar, Chile)

\begin{tabular}{lcc}
\hline Característica & $\mathrm{n}$ & $\%$ \\
\hline Género & & \\
$\quad$ Mujer & 29 & 58 \\
$\quad$ Hombre & 21 & 42 \\
Edad (años) & & \\
$\quad<25$ & 6 & 12 \\
$26-50$ & 36 & 72 \\
$\quad>51$ & 8 & 16 \\
Nivel de enseñanza & & \\
Básica completa & 1 & 2 \\
Media incompleta & 1 & 2 \\
Media completa & 7 & 14 \\
Técnica incompleta & 2 & 4 \\
Técnica completa & 7 & 14 \\
Universitaria & 12 & 24 \\
incompleta & & \\
Universitaria & 20 & 40 \\
completa & & \\
\hline
\end{tabular}

- ¿ ¿Razón de tenencia?: Compañía 38\% y seguridad 6\%;

- $\quad$ ¿Tiempo con la mascota?: $38 \%$ entre 2 y 6 años, 34\% más de 6 años;

- ¿Cómo llegó a su poder?: Compra 46\%, regalo $36 \%$;

- $\quad$ ¿Tiene implantado el microchip?: 58\% No, donde $34 \%$ por desconocimiento y $34 \%$ no sabe/no responde;

- ¿Dónde vive la mascota?: $40 \%$ en el patio, 38\% dentro de la casa;

- ¿Cuántas mascotas caninas tiene a su cargo?: 38\%:1;32\%: 2 .

Al consultar sobre la relación existente con sus mascotas, solicitando la opción que mejor la definía, se obtuvieron los siguientes resultados: Responsable (46\%), dueño (34\%), propietario $(10 \%)$, tenedor $(4 \%)$, amigo $(2 \%)$, familia $(2 \%)$ y otra $(2 \%)$. No se encontró asociación significativa entre las variables 


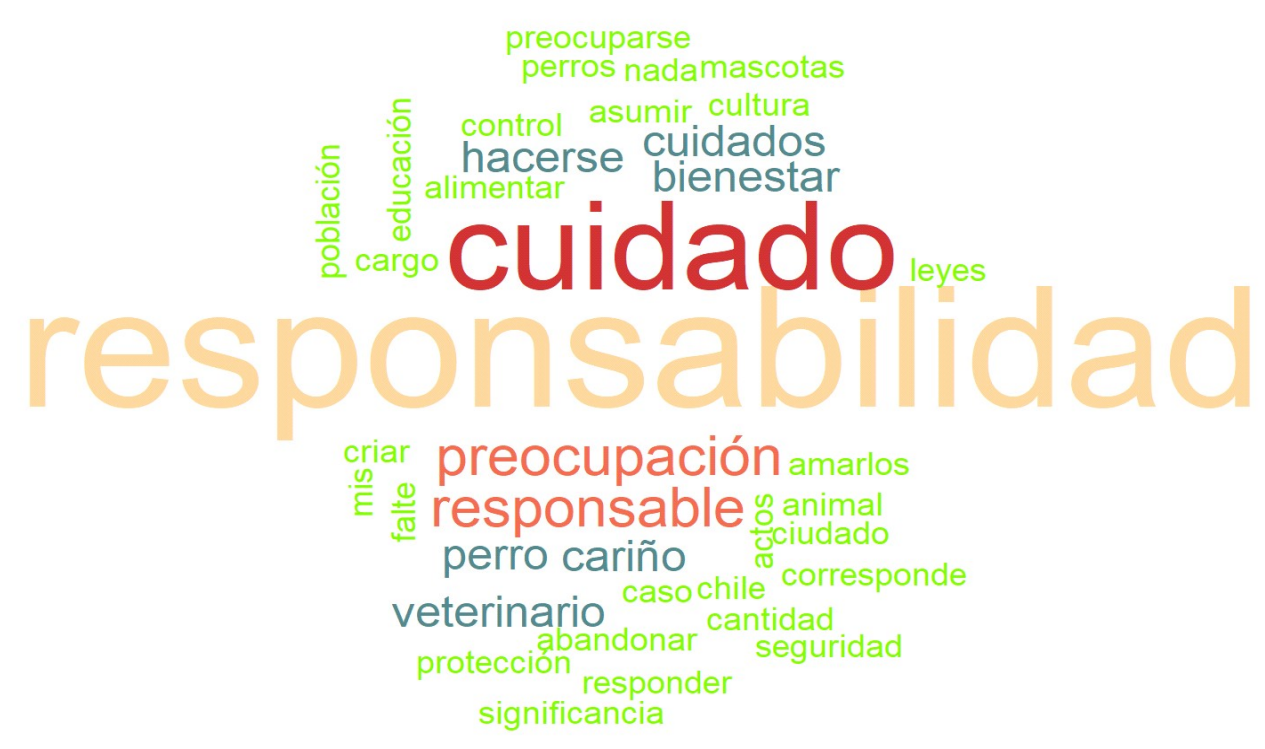

Figura 1. Imagen espontánea sobre tenencia responsable de mascotas

categóricas, características sociodemográficas y valoraciones sobre tenencia responsable

Frente a la pregunta de respuesta libre (imagen espontánea), Cuando hablo de tenencia responsable ¿Qué viene a su mente?, se obtuvo que las mayores frecuencias correspondieron a las palabras responsabilidad y cuidado. En la Figura 1 se presenta la nube de palabras sobre la valoración de los encuestados.

En la caracterización de la salud reproductiva, con posibles respuestas entre muy en desacuerdo hasta muy de acuerdo, destacaron las siguientes respuestas:

- La castración de los perros constituye un buen método para el control de la población canina: $84 \%$ estuvo de acuerdo o muy de acuerdo.

- Castrar a un perro es dañino para su salud en general: $58 \%$ estuvo en desacuerdo o muy en desacuerdo.
- Castrar a un perro afecta su sexualidad: $60 \%$ estuvo en desacuerdo o muy en desacuerdo.

- Para el control de la población canina es más efectivo esterilizar hembras que machos: $46 \%$ estuvo en desacuerdo o muy en desacuerdo.

Ante la consulta ¿Su mascota se ha reproducido/tiene descendencia/tiene crías?, 32 (64\%) tenedores indicaron que no $(64 \%)$. De otra parte, de los 18 que señalaron la opción «Sí̀», 2 perros eran mestizos y 16 de raza, todos mayores de 2 años. No se encontró asociación significativa entre las variables categóricas, características sociodemográficas y valoraciones sobre salud reproductiva.

Ante la pregunta de respuesta libre (imagen espontánea), Cuando hablo de salud reproductiva en perros ¿Qué viene a su mente?, las mayores frecuencias correspondieron a las palabras veterinario, salud y cuidado, tal como se aprecia en la nube de palabras presentada en la Figura 2. 


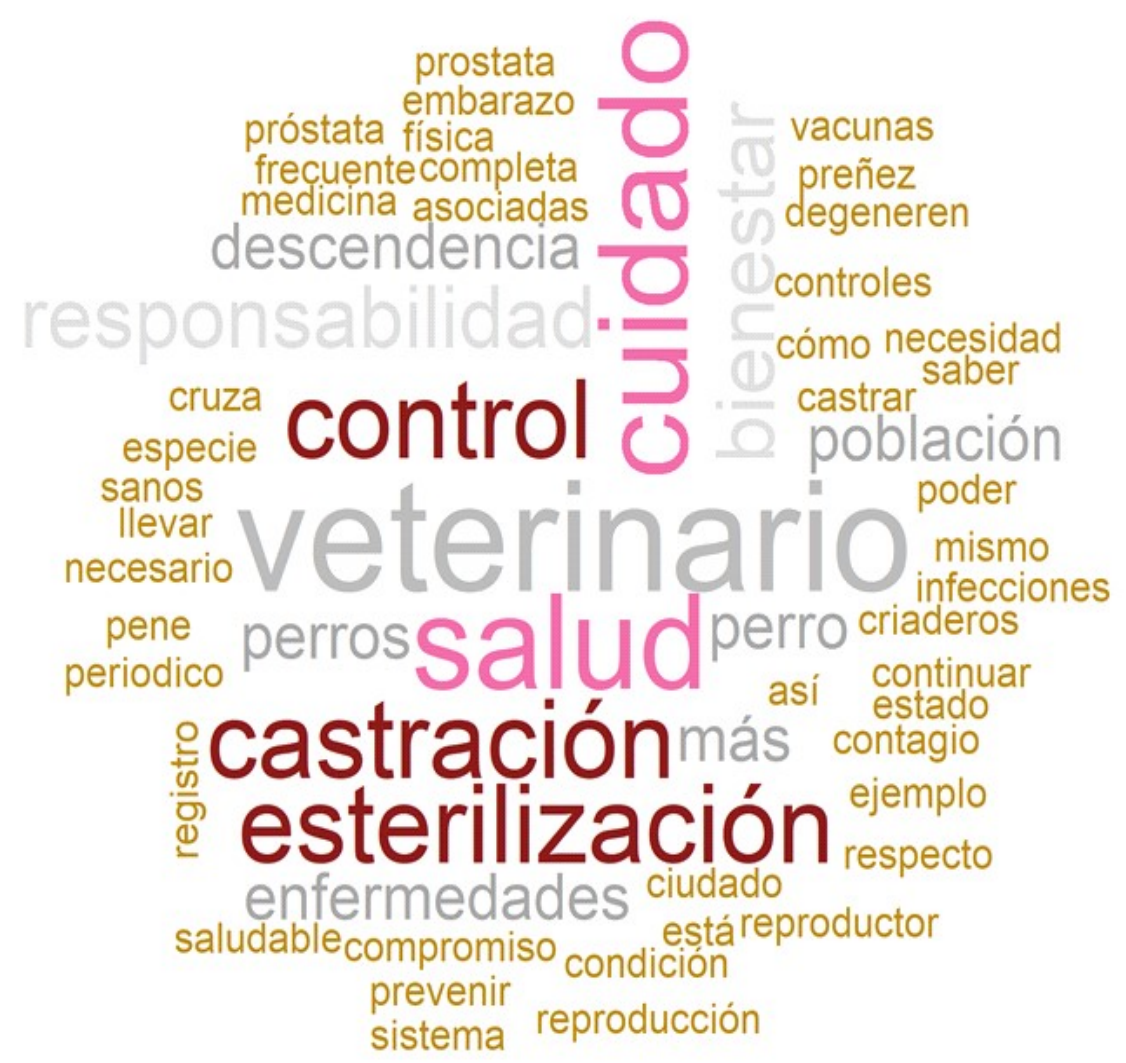

Figura 2. Imagen espontánea sobre salud reproductiva en perros

\section{Discusión}

Los resultados de la evaluación de salud reproductiva fueron concordantes con varias descripciones que señalan un incremento de anormalidades prostáticas y testiculares subclínicas en perros enteros mayores a cinco años (Polisca et al., 2016; Bhanmeechao et al., 2018; Sánchez et al., 2019; Sánchez y Pfeffer, 2020).

Estos hallazgos podrían ser atribuidos a la actividad testicular, a través de la secreción de testosterona, la cual es considerada un factor endocrino relevante para el desarrollo de procesos patológicos en próstata, como sería el caso de la hiperplasia prostática benigna, condición que además se asocia positivamente con la edad (Root Kustritz, 2014;
Christensen, 2018). Además, las anomalías que pueden afectar el tono testicular, como la degeneración testicular se describen con frecuencia en perros mayores de cinco años (Bhanmeechao et al., 2018). Por otra parte, la citología prepucial denotó normalidad en todos los canes, lo cual se podría asociar a que no se observaron casos de neoplasias testiculares (Sánchez, 2020).

En el presente estudio, se prestó atención especial a perros adultos enteros, con el propósito de caracterizar a los tenedores y poder educar al público general de los beneficios de la esterilización para la salud y el bienestar animal. Así como también, presentar una propuesta a los médicos veterinarios respecto del manejo preventivo en salud reproductiva de perros enteros (Sánchez, 2018). 
En relación con los tenedores, llamó la atención que en la mayoría de los ejemplares no se tenía como propósito la reproducción y que el mayor porcentaje de los encuestados consideró que la castración de perros es un buen método para el control de población. De otra parte, es importante destacar que, entre los hábitos de tenencia de mascotas en la ciudad de Viña del Mar, así como probablemente en otras ciudades de Latinoamérica, existe un alto porcentaje de perros enteros (Morales et al., 2009).

En general se puede señalar que el conocimiento y la actitud respecto de los temas de tenencia responsable y salud reproductiva fue positiva; sin embargo, en la práctica se observan algunas contradicciones, las cuales no se pueden adjudicar a algún aspecto de las características sociodemográficas analizadas en el estudio. Es sí que si las campañas relacionadas con la tenencia de animales de compañía se centran en el concepto de propiedad responsable, se podría inferir que falta mayor profundidad y coherencia en cómo se educa a la población.

Se ha observado que lo que parece ser una descripción objetiva de las prácticas de tenencia responsable de perros, que debieran ser fáciles de seguir, son en realidad una compleja interacción de creencias basadas en cuestiones de prácticas éticas, en intereses percibidos y en la naturaleza de la relación social con el perro. Agregando, que la tenencia responsable de un perro significa supuestos distintos para las diferentes personas, en diversos momentos (Westgarth et al., 2019).

En este sentido resulta interesante destacar que la mayoría de los tenedores encuestados señaló que el concepto que mejor definía la relación con su perro era la de responsable. Además, en la percepción libre de tenencia responsable el concepto más reiterado fue también la responsabilidad. Esto debería ser considerado para que los progra- mas de educación funcionen, ya que hablarles de responsabilidad no tendría un gran impacto puesto que los tenedores ya creen que son responsables (Westgarth et al., 2019). Al respecto, es importante considerar que el afecto y la emoción son fundamentales para la percepción de la responsabilidad (Lerner et al., 1998; Nichols y Knobe, 2007).

\section{Conclusiones}

- Las afecciones reproductivas subclínicas son comunes en perros adultos enteros, poniendo en evidencia una merma en la salud y, por ende, en el bienestar animal.

- No existe asociación significativa entre el conocimiento y la actitud en relación con los tópicos de tenencia responsable y salud reproductiva con las características sociodemográficas.

- Los encuestados manifestaron tener una actitud a favor de la tenencia responsable de mascotas, lo que se contrapone con la práctica, de allí, emerge el desafío de una educación integral, con una mayor profundidad.

\section{Literatura Cittada}

1. Bhanmeechao C, Srisuwatanasagul S, Ponglowhapan. 2018. Age-related changes in interstitial fibrosis and germ degeneration of the canine testis. Reprod Domest Anim 53: 37-43. doi: 10.1111/ rda.13354

2. Caron-Lormier G, England G, Green M, Asher L. 2016. Using the incidence and impact of health conditions in guide dogs to investigate healthy ageing working does. Vet J 207: 124-130. doi: 10.1016/j.tvj1.2015.10.046

3. Christensen B. 2018. Canine prostate disease. Vet Clin North Am Small Anim Pract 48: 701-709. doi: 10.1016/j.cvsm.2018.02.012 
4. Dawkins M. 2007. Through animal eyes: what behaviour tell us. Appl Anim Behav Sci 100: 4-10.

5. Lerner J, Goldberg J, Tetlock P. 1998. Sober second thought: the effects of accountability, anger, and authoritarianism on attributions of responsibility. Pers Soc Psychol Bull 24: 563-574. doi: 10.1177/0146167298246001

6. Meyers-Wallen V. 2012. Application of genome and molecular methods to fundamental questions in canine and feline reproductive health. Reprod Dom Anim 47 (Suppl 6): 309-312. doi: 10.1111/ rda. 12043

7. Ministerio de Salud. 2017. Ley 21020 Sobre Tenencia Responsable de Mascotas y Animales de Compañía. Chile. [Internet]. Disponible en: http://bcn.cl/ 2902q

8. Morales M, Varas C, Ibarra L. 2009. Caracterización demográfica de la población de perros de Viña del Mar, Chile. Arch Med Vet 41: 89-95. doi: 10.4067/ S0301-732X2009000100013

9. Nichols S. Knobe J. 2007. Moral responsibility and determinism: the cognitive science of folk intuitions. Noûs 41: 663-685. doi: 10.1111/j.14680068.2007.00666.x

10. Nicks B, Vandenheede M. 2014. Santé et bien-être des animaux: équivalence ou complémentarité? Rev Sci Tech Off Int Epiz 33: 91-96.

11. [OIE] Organización Mundial de Sanidad Animal. 2013. Prevención y control de las enfermedades animales. Hojas informativas. [Internet]. Disponible en: https://www.oie.int/fileadmin/Home/ esp/Media_Center/docs/pdf/Fact_sheets/P-C_ES.pdf

12. Polisca A, Troisi A, Fontain E, Menchetti L, Fontbonne 2016. A retrospective study of canine prostatic disease from 2002 to 2009 at the Alfort Veterinary College in France. Theriogenology 85: 835-840. doi: 10.1016/ j.theriogenology.2015.10.030

13. Root Kustritz M. 2014. Applied small animal andrology. In. Chenoweth P, Lorton S (eds). Animal andrology: theories and applications. USA: CAB International. p 177-197.

14. Sánchez A. 2018. Salud reproductiva del macho canino. Invest Cienc Anim 2: 33-36.

15. Sánchez, A, Fierro C, Troya F, Pfeffer M. 2019. Caracterización de salud reproductiva en perros. Rev Inv Vet Perú 30: 1249-1256. doi: 10.15381/rivep.v30i3.16723

16. Sánchez A. 2020. Caracterización de la citología prepucial en perros adultos. Rev Vet 31: 89-91.

17. Sánchez A, Pfeffer M. 2020. Evaluación de salud reproductiva en perros machos mestizos enteros. Rev Vet 31: 206-209.

18. Villamil L, Romero J. 2003. Retos y perspectivas de la salud pública veterinaria. Rev Salud Pública 5: 109-122.

19. Westgarth C, Christley R, Marvin G, Perkins E. 2019. The responsible dog owner: The construction of responsibility. Anthrozoös 32: 631-646. doi: 10.1080/ 08927936.2019.1645506 